\title{
Sicherungsverwahrung (erneut) auf dem Prüfstand
}

Frieder Dünkel

\author{
Anmerkungen zu zwei Entscheidungen des Bundesverfassungsgerichts - BVerfG - 2 BvR \\ 2029/01 v. 5.2.2004 und BVerfG - 2 BvR 834/02 - 2 \\ BvR 1588/02 v. 10.2.2004 und zum Referentenentwurf des Bundesministeriums der Justiz \\ zur Einführung der nachträglichen Sicherungsverwahrung vom 9.3.2004
}

\section{Vorgeschichte}

$\mathrm{D}$ ie Sicherungsverwahrung ist neben der lebenslangen Freiheitsstrafe die schärfste Sanktion des deutschen Strafrechts und als Maßregel der Besserung und Sicherung von unbestimmter Dauer, potenziell bis zum Lebensende des Verurteilten. Sie wird neben einer Freiheitsstrafe bei als gefährlich eingeschätzten Straftätern (vgl. zu den Voraussetzungen im einzelnen $\S 66$ StGB) angeordnet und im Anschluss an die voll verbüßte Freiheitsstrafe vollstreckt (vgl. § 67 StGB). Historisch geht sie auf das Gewohnheitsverbrechergesetz der Nationalsozialisten aus dem Jahr 1933 zurück, wurde aber nach Ende des zweiten Weltkrieges beibehalten. Im Zuge der Strafrechtsreformgesetze der 1960er und 1970er Jahre war die Sicherungsverwahrung stark kritisiert worden, weshalb 1969 die formellen und materiellen Voraussetzungen der Sicherungsverwahrung restriktiver gefasst wurden. Dementsprechend gelang es, ihre Anwendungspraxis stark einzuschränken. Wurden in den 1960er Jahren noch jährlich etwas mehr als 200 Personen zur Sicherungsverwahrung verurteilt, so in den 1980er bis Anfang der 1990er Jahre nur ca. 40 oder weniger Täter (1990: 31). Die Stichtagsbelegung im Strafvollzug ging dementsprechend von ca. 1.500 Anfang der 1960er Jahre auf weniger als 200 in den 1980er Jahren zurück. Das entspricht ca. 0,3\% der gesamten Population des Strafvollzugs. Seit einigen Jahren, insbesondere seit den Verschärfungen durch das Gesetz zur Bekämpfung von Sexualdelikten und anderen gefährlichen Straftaten vom
26.1.1998, sind die jährlichen Verurteilungen auf 74 im Jahr 2001 und eine Stichtagsbelegung von 230 (31.3.2003) angestiegen. Das Gesetz von 1998 ist im Zusammenhang mit einigen gravierenden Einzelfällen von Sexualstraftaten zu sehen, die, nicht zuletzt vor dem Hintergrund des Falles Dutroux in Belgien, erhebliches Aufsehen erregten und die dazu führten, dass die Möglichkeiten der Anordnung von Sicherungsverwahrung auch ohne entsprechende Vorverurteilungen in $\S 66$ Abs. 3 StGB erweitert wurden (vgl. i. e. Dünkel 2004). Im Jahr 2002 wurde schließlich die nachträgliche Anordnung einer (zuvor vorbehaltenen) Sicherungsverwahrung (s.u.) erlaubt.

Die beiden zu besprechenden Entscheidungen des BVerfG stehen in engem Zusammenhang mit der Reform von 1998 und einer weiteren Gesetzesreform vom 21.8.2002, im Rahmen derer die nachträgliche Anordnung der Sicherungsverwahrung in Fällen ermöglicht wurde, bei denen sich erst während des Vollzugs die Gefährlichkeit des Gefangenen herausstellt (vgl. § 66a StGB; BGBl. I, S. 3344 ff.). Die rot-grüne Regierungskoalition hatte auf den Druck der Opposition reagiert, die eine noch weitergehendere Möglichkeit der nachträglichen Sicherungsverwahrung schaffen wollte (s. u.). § 66a StGB lässt eine nachträgliche Anordnung nur zu, wenn zum Zeitpunkt des Urteils die formalen Voraussetzungen des $\S 66$ StGB vorliegen und im Ausgangsurteil bereits die nachträgliche Anordnung ausdrücklich vorbehalten wurde. Die konservativ regierten Bundesländer wie Bayern, Baden-Württemberg, Hessen oder Sachsen-Anhalt hatten seit 1998 mehrere Gesetzesinitiativen im Bundestag bzw. Bundesrat eingebracht, mit denen eine nachträgliche Sicherungsverwahrung auch ohne vorherigen Vorbehalt eingeführt werden sollte. Nachdem alle entsprechenden Gesetzesanträge im Bundestag oder Bundesrat abgelehnt worden waren, gingen einige Bundesländer dazu über, die Materie polizeirechtlich zu definieren und die angebliche "Sicherheitslücke« durch eigenständige Landesgesetze (sog. Straftäterunterbringungsgesetze) $\mathrm{zu}$ schließen. Dieser politische Konflikt stand in der zweiten Entscheidung vom 10.2.2004 zur Debatte. Juristischer Anknüpfungspunkt war die Frage, ob die Länder hierfür eine eigene Gesetzgebungskompetenz haben oder nicht. Handelt es sich um Fragen der polizeilichen Gefahrenabwehr, so die Behauptung der entsprechenden Länder (und im übrigen auch die seinerzeitige Rechtsauffassung des Bundesjustizministeriums bzw. der Bundesregierung in ihrer Stellungnahme zu den entsprechenden Verfassungsbeschwerden), so hätten diese eine originäre Gesetzgebungskompetenz (vgl. Art. 70 Abs. 1 GG). Gehört die nachträgliche Sicherungsverwahrung dagegen zum Bereich des Strafrechts, so ist gem. Art. 74 Abs. 1 Nr. 1 ein Fall der konkurrierenden Gesetzgebungskompetenz gegeben. Wenn der Bundesgesetzgeber den Sachbereich umfassend und abschließend geregelt hat (was nach Auffassung der Beschwerdeführer mit der Einführung des $\S$ 66a StGB der Fall war), verbleibt für den Landesgesetzgeber gem. Art. 72 Abs. 1 GG keine weitere Gesetzgebungskompetenz.

Hintergrund der Entscheidung vom 5.2.2004 war die Neuregelung der Sicherungsverwahrung im er- wähnten Reformgesetz von 1998. Vor 1998 war die Sicherungsverwahrung bei der erstmaligen Anordnung auf maximal 10 Jahre begrenzt (vgl. § 67d Abs. 1 StGB a. F.). Mit der Reform wurde diese 10-Jahresfrist aufgehoben und stattdessen lediglich eine besonders sorgfältige Prüfung nach Ablauf von 10 Jahren in $\S 67 d$ Abs. 3 StGB gesetzlich vorgeschrieben. Zugleich wurden die Kriterien für eine Entlassung in $\S 67 d$ Abs. 2 StGB verschärft. Vor 1998 genügte, dass verantwortet werden konnte, zu erproben, dass der Verurteilte keine weiteren Straftaten mehr begehen werde, womit ein gewisses "Restrisiko" in Kauf genommen wurde. Dieses war nach der Rechtsprechung je nach den zu erwartenden Straftaten zu differenzieren, d. h. es konnte bei lediglich zu erwartenden Bagatelltaten eine Entlassung eher verantwortet werden (vgl. i. e. Nomos Kommentar-Dünkel, § 57 Rn. 14 ff.). Die neue Formulierung von 1998 ersetzte die Erprobungsklausel durch eine sog. Erwartungsklausel: eine Aussetzung (und damit Entlassung aus der Sicherungsverwahrung) soll nur erfolgen, »wenn zu erwarten ist, dass der Untergebrachte außerhalb des Maßregelvollzugs keine rechtswidrigen Taten mehr begehen wird « $(\S 67 \mathrm{~d}$ Abs. 2 StGB).

Verfassungsrechtliche Streitfrage war hier, ob diese Verschlechterungen aus der Sicht der betroffenen Untergebrachten bei derzeit im Strafvollzug einsitzenden Sicherungsverwahrten gegen das Rückwirkungsverbot des $\S 103$ Abs. 2 GG bzw. den aus dem Rechtsstaatsprinzip abzuleitenden Vertrauensschutzgedanken verstoßen und ob der Gesetzgeber aus verfassungsrechtlichen Gründen (insbesondere des Schutzes der Menschenwürde) nicht zur Schaffung einer gesetzlich geregelten Obergrenze der Sicherungsverwahrung verpflichtet ist.

\section{Inhalt der beiden Entscheidungen des BVerfG}

Die beiden Entscheidungen sind von herausragender Bedeutung, nicht nur, weil sie Grundfragen der Gesetzgebungskompetenzen und des Rückwirkungsverbots im Strafrecht im Hinblick auf Maßregeln der Besserung und Sicherung klären, son- 
dern auch, weil sie grundlegende verfassungsrechtliche Vorgaben für die Ausgestaltung der Sicherungsverwahrung machen und damit den expansiven Bestrebungen eines angesichts vermeintlicher Sicherheitslücken immer weiter expandierenden Sicherheitsstrafrechts und Sicherheitsstrafvollzugs Grenzen setzen.

Während im ersten Fall die Verfassungsbeschwerde erfolglos blieb, aber dennoch einige wichtige verfassungsrechtliche Klarstellungen erfolgten und die in einigen früheren Entscheidungen (insbesondere aus dem Jahr 1998) entwickelten Leitlinien zur Resozialisierung als aus dem Schutz der Menschenwürde und dem Sozialstaatsprinzip gefolgerten Verfassungsgebot fortgeführt wurden (vgl. III.), waren die Verfassungsbeschwerden im zweiten Fall weitgehend erfolgreich (vgl. IV.). Allerdings erscheint hierbei die auch innerhalb des Gerichts umstrittene Entscheidung bemerkenswert, dass trotz der Verfassungswidrigkeit der Landesgesetze diese bis zum 30.9.2004 anwendbar bleiben, weshalb die entsprechenden Beschwerdeführer auch in Haft blieben. Das BVerfG wollte hiermit dem Bundesgesetzgeber Gelegenheit geben, eine bundesgesetzliche Regelung zu schaffen, die den Bedürfnissen der Länder entspricht. Ob diese allerdings jemals mit den verfassungsrechtlichen Vorgaben in Einklang $\mathrm{zu}$ bringen sein werden, bleibt fraglich (vgl. hierzu V.).

\section{Leitsätze und Gründe}

Die Entscheidung vom 5.2.2004 hat folgende Leitsätze:

1.a) Die Menschenwürde wird auch durch eine langdauernde Unterbringung in der Sicherungsverwahrung nicht verletzt, wenn diese wegen fortdauernder Gefährlichkeit des Untergebrachten notwendig ist. Erforderlich ist aber auch in diesen Fällen, die Eigenständigkeit des Untergebrachten zu wahren, seine Würde zu achten und zu schützen. Daher muss die Sicherungsverwahrung ebenso wie der Strafvollzug darauf ausgerichtet sein, die Voraussetzungen für ein verantwortliches Leben in Freiheit zu schaffen. b) Für das Institut der Sicherungsverwahrung folgt aus Art. 1 Abs. 1 GG kein verfassungsrechtliches Gebot, schon bei der Anordnung der Sicherungsverwahrung oder in einem späteren Überprüfungszeitpunkt eine Höchstfrist des Vollzuges festzusetzen. Es ist nicht zu beanstanden, wenn der Gesetzgeber vorsieht, dass eine verbindliche Entscheidung über den voraussichtlichen Entlassungszeitpunkt beim Sicherungsverwahrten nicht im Vorhinein getroffen wird.

2.a) Je länger die Unterbringung in der Sicherungsverwahrung andauert, umso strenger sind die Voraussetzungen für ihre Fortdauer.

b) Die Vorschrift des $\S 67 d$ Abs. 3 StGB trägt der verstärkten Geltung des Freiheitsanspruchs nach zehnjähriger Verwahrungsdauer Rechnung, indem sie erhöhte Anforderungen an das bedrohte Rechtsgut und den Nachweis der Gefährlichkeit des Verwahrten stellt und nur ausnahmsweise die Fortsetzung der Vollstreckung gestattet.

c) Wegen der besonderen Bedeutung der Vollzugslockerungen für die Prognosebasis darf sich das Vollstreckungsgericht nicht damit abfinden, dass die Vollzugsbehörde ohne hinreichenden Grund Vollzugslockerungen versagt, welche die Erledigung der Maßregel vorbereiten können.

3. Der Anwendungsbereich von Art. 103 Abs. 2 GG ist auf staatliche Maßnahmen beschränkt, die eine missbilligende hoheitliche Reaktion auf ein rechtswidriges, schuldhaftes Verhalten darstellen und wegen dieses Verhaltens ein Übel verhängen, das dem Schuldausgleich dient.

4. Der Wegfall der Höchstfrist für eine erstmalig angeordnete Sicherungsverwahrung und die Anwendbarkeit auf Straftäter, bei denen die Sicherungsverwahrung vor Verkündung und Inkrafttreten der Novelle angeordnet und noch nicht erledigt war, steht in Einklang mit dem rechtsstaatlichen Vertrauensschutzgebot (Art. 2 Abs. 2 GG in Verbindung mit Art. 20 Abs. 3 GG).
Bei dem 1957 geborenen Beschwerdeführer handelt es sich um einen Straftäter, der sein Leben seit dem 15. Lebensjahr fast ausschließlich in Haft verbracht hat, zunächst wegen Eigentumsdelikten, später wegen Raub, gefährlicher Körperverletzung und schließlich wegen versuchten Mordes und Raubes. Immer wieder wurde er auch in der Haft gewalttätig und deshalb zu weiteren Freiheitsstrafen verurteilt. Die letzte Verurteilung lautete im Jahr 1986 auf fünf Jahre Freiheitsstrafe mit anschließender Sicherungsverwahrung. Nach altem Recht wäre der Beschwerdeführer deshalb 2001 aus der Sicherungsverwahrung zu entlassen gewesen. Während des Vollzugs der Sicherungsverwahrung fiel der Beschwerdeführer durch impulsiv-aggressives Verhalten auf (u. a. brach er einem Mitgefangenen aus nichtigem Anlass das Nasenbein) und begann sich zu der rechtsextremen Skinheadszene zu bekennen. Mehrere psychiatrische Gutachten kamen zum Schluss, dass von der weiteren Gefährlichkeit auszugehen und deshalb die weitere Unterbringung notwendig sei.

Das BVerfG stellt einleitend fest, dass die Unterbringung in der Sicherungsverwahrung keine die Menschenwürde verletzende grausame, unmenschliche oder erniedrigende Strafe darstellt, wenn gewährleistet wird, dass der Untergebrachte eine realistische Chance behält, »je wieder der Freiheit teilhaftig zu werden (vgl. schon BVerfGE 45, 187, 229 in der grundlegenden Entscheidung zur lebenslangen Freiheitsstrafe, vgl. hierzu van Zyl Smit, Taking Life Imprisonment Seriously, 2002, S. 145 ff.). Ebenso wie für die lebenslange Freiheitsstrafe fordert das BVerfG, dass auch die Sicherungsverwahrung nur dann verfassungsgemäß ist, wenn ein sinnvoller Behandlungsvollzug vorgesehen wird, der schädlichen Auswirkungen des Freiheitsentzugs entgegen wirkt. Der Staat dürfe sich zwar gegenüber gefährlichen Straftätern durch Freiheitsentzug sichern, jedoch müsse auch in der Sicherungsverwahrung auf eine Resozialisierung des Untergebrachten hingewirkt werden (vgl. Leitsatz 1a). Diesem Maßstab genüge die Sicherungsverwahrung in ihrer derzeitigen Ausgestaltung. Das Gericht hatte sich hinsichtlich der geltenden Haftbedingungen durch eine
Umfrage bei den Landesjustizverwaltungen und die Anhörung von Vollzugspraktikern sachkundig gemacht. Die Sicherungsverwahrung sei damit normativ wie tatsächlich am Resozialisierungsgedanken ausgerichtet und in der Praxis nicht als reiner Verwahrvollzug ausgestaltet. Allerdings kritisiert das BVerfG, dass die Landesjustizverwaltungen nicht über einheitliches statistisches Material zur Vollzugspraxis verfügten und fordert daher eine regelmäßige nachvollziehbare »Überprüfung «, dass die in der Sicherungsverwahrung Untergebrachten allgemein nicht nur rechtlich, sondern auch tatsächlich eine realistische Chance haben, die Freiheit wieder $\mathrm{zu}$ erlangen. Dies schließt Erhebungen darüber ein, ob den Sicherungsverwahrten hinreichende Resozialisierungsangebote, insbesondere Behandlungs-, Therapie- oder Arbeitsmöglichkeiten angeboten werden.«

Eine vorherige Festsetzung der Höchstdauer der Sicherungsverwahrung sei verfassungsrechtlich nicht geboten (vgl. Leitsatz 1b). Die Zehnjahres-Höchstfrist bei der erstmaligen Unterbringung existierte im übrigen historisch gesehen erst seit der Strafrechtsreform von 1975. Das System wiederkehrender Überprüfungen von Aussetzungs- und Erledigungsreife gewährleistet nach Auffassung des BVerfG »die angemessene prozedurale Rechtssicherheit.« Im übrigen sei hierbei von Bedeutung, dass die Neuregelung des $§ 67 \mathrm{~d}$ Abs. 3 StGB die Weitervollstreckung der Sicherungsverwahrung über 10 Jahre hinaus nur ausnahmsweise gestatte.

Das BVerfG bewahrt im übrigen bei der Einschätzung der kriminalpolitischen Geeignetheit oder Notwendigkeit der Verschärfungen der Sicherungsverwahrung die ihm zukommende Zurückhaltung, indem es betont, dass der Gesetzgeber insoweit einen Beurteilungsspielraum habe, der nur eingeschränkter Überprüfung zugänglich sei.

Das Spannungsverhältnis zwischen dem Freiheitsanspruch des Untergebrachten und dem Sicherungsbedürfnis der Allgemeinheit verlange mit zunehmender Vollzugsdauer strengere Voraussetzungen für die Verhältnismäßigkeit des Freiheitsentzugs (vgl. Leitsätze 2a) und 
b), die im Einzelfall abzuwägen sind (vgl. BVerfGE 70, 297, 311; auch hier werden die Grundsätze zur Verfassungsmäßigkeit der lebenslangen Freiheitsstrafe übernommen). Dies komme u. a. dadurch zum Ausdruck, dass das Regel-Ausnahmeverhältnis von Aussetzung und Fortdauer des $\S 67 d$ Abs. 2 in $\S 67 d$ Abs. 3 StGB zugunsten der regelmäßigen Aussetzung nach 10 Jahren umgekehrt worden sei. Das Gesetz gehe davon aus, »dass sich die Gefährlichkeit nach der rein spezialpräventiven Ausrichtung des Maßregelvollzugs der Sicherungsverwahrung seien - bei lang andauernder Unterbringung zusätzliche - Vergünstigungen für die Untergebrachten vorzusehen, »um dem hoffnungslos Verwahrten einen Rest an Lebensqualität zu gewährleisten.«

Die verfassungsrechtlich besonders brisante Frage betraf die Frage des Rückwirkungsverbots i. S. d.

Die Unterbringung in der Sicherungsverwahrung stellt keine die Menschenwürde verletzende grausame, unmenschliche oder erniedrigende Strafe dar, wenn gewährleistet wird, dass der Untergebrachte eine realistische Chance behält, "je wieder der Freiheit teilhaftig zu werden" (BVerfG)

Ablauf von zehn Jahren regelmäßig erledigt « habe (vgl. hierzu Streng in Festschrift für Lampe, 611, $633 \mathrm{~m}$. w. N.). Damit verbietet sich die schlichte Fortschreibung unwiderlegter Gefährlichkeitshypothesen und es werden konkrete, gegenwärtige Anhaltspunkte für die weitere Gefährlichkeit verlangt. Zweifel gehen nicht zu Lasten des Untergebrachten, nur im Falle der Widerlegung der nach 10 Jahren vermuteten Ungefährlichkeit darf die weitere Unterbringung erfolgen (dies gilt im übrigen auch für die wiederholte Unterbringung in der Sicherungsverwahrung, womit die Neuregelung das frühere Recht sogar abgemildert hat, vgl. § 67d Abs. 1 StGB a. F.).

Im Anschluss an einige frühere Entscheidungen (vgl. BVerfG NJW 1998, 1133, 1134; NStZ 1998, 373, 374) hebt das BVerfG die Bedeutung von Vollzugslockerungen als Voraussetzung für eine wissenschaftlich fundierte Prognoseeinschätzung hinsichtlich des zukünftigen Legalverhaltens hervor. Da sie notwendige Entscheidungsgrundlage für die Prognoseentscheidung seien, dürfe die Vollzugsbehörde Lockerungen nicht ohne hinreichenden Grund versagen. Im übrigen finden sich interessante Hinweise für die Vollzugsgestaltung: wegen gleichfalls nicht dem Rückwirkungsverbot unterliegen)

Abschließend wird festgestellt, dass auch der übergeordnete, aus dem Rechtsstaatsprinzip abzuleitende Vertrauensschutzgedanke nicht verletzt ist (vgl. Leitsatz 4). Das auf Strafen bezogene Rückwirkungsverbot des Art. 103 Abs. 2 GG bedeute nicht, dass damit in allen anderen Bereichen Rückwirkungen unbedenklich seien. Der Bürger müsse sich auf die Verlässlichkeit der Rechtsordnung verlassen können, weshalb Änderungen, die diesen Vertrauensschutz tangieren, einer besonderen Rechtfertigung bedürfen. Dabei unterscheidet das BVerfG zwischen der sog. echten Rückwirkung, bei der eine Rückbewirkung von Rechtsfolgen an einen Tatbestand vor Erlass des neuen Gesetzes erfolgt, und der sog. unechten Rückwirkung, die zwar eine tatbestandliche Rückanknüpfung beinhaltet, jedoch treten die Rechtsfolgen erst nach Verkündung der Norm ein. Der Wegfall der Zehnjahres-Höchstfrist wirkt nicht auf einen Zeitpunkt vor dem Inkrafttreten der Reform zurück, ist also eine solche »unechte « Rückwirkung, die den Vertrauensschutz weniger stark beeinträchtigt als eine echte Rückwirkung. Gleichwohl war mit der absoluten Höchstfrist die Erwartungshaltung des Untergebrachten gegeben, spätestens nach 10 Jahren mit Sicherheit entlassen zu werden. Diese Erwartungshaltung steht allerdings unter dem Gesetzesvorbehalt des § 2 Abs. $6 \mathrm{StGB}$, wonach für die Anordnung wie Vollstreckung von Maßregeln das zur Zeit der Entscheidung geltende Recht gilt. Dennoch bedeutet dies nicht automatisch den Vorrang des Interesses des Gesetzgebers vor dem Vertrauensschutz des Untergebrachten auf den unveränderten Bestand der Norm, hier des § 67d Abs. 1 StGB a. F. Vielmehr bedarf es einer Abwägung, die das BVerfG hier zugunsten der Sicherheitsinteressen der Allgemeinheit vornimmt. Die "Schutzinteressen der potenziellen Opfer vor potenziellen Straftätern « sind umso stärker zu berücksichtigen, je existenzieller die bedrohten Grundrechte der möglichen Opfer sind. Erneut betont das BVerfG, dass es ihm nicht zustehe, zu hinterfragen, ob die kriminalpolitische Entscheidung der Aufhebung der Höchstfrist gebo- ten war. Die Annahme, dass auf diese Weise potenzielle Opfer besser geschützt werden können, unterliegt jedenfalls keinen verfassungsrechtlichen Bedenken, da der Gesetzgeber im Rahmen seines Gestaltungsspielraums gehandelt und insbesondere nicht gegen das Übermaßverbot verstoßen habe. Die Entscheidung erging hinsichtlich der Rückwirkungsfrage mit $6: 2$ Stimmen, im übrigen einstimmig. Die abweichenden Voten sind bislang nicht zugänglich.

Die Entscheidung verdient im Ergebnis und in der Begründung Zustimmung. Ihre Bedeutung geht weit über die unmittelbar verfassungsrechtlichen Fragen hinaus, weil das BVerfG sich erneut veranlasst sah, Grundprinzipien von Strafen und Maßregeln sowie ihres Vollzugs zu verdeutlichen, die angesichts einer den Sicherheitsgedanken einseitig interpretierenden Kriminalpolitik verloren zu gehen drohen. So z. B. die Einsicht, dass auch bei Sicherungsverwahrten nicht das schlichte und dauerhafte Wegschließen, sondern ein konsequenter Resozialisierungsvollzug am besten die Sicherheit der Allgemeinheit schützen kann. Die Annahme, dass die derzeitige Ausgestaltung der Sicherungsverwahrung resozialisierungsorientiert sei, stützt sich allerdings auf - wie auch vom BVerfG moniert - unzulänglich und unsystematisch belegte Selbstauskünfte einzelner Landesjustizverwaltungen. Zu verlangen wäre angesichts der bei langiähriger Inhaftierung $\mathrm{zu}$ besorgenden negativen Wirkungen der Haft mindestens ein Standard wie er in den sozialtherapeutischen Anstalten und Abteilungen üblich ist. Dies wird man vom derzeitigen Vollzug der Sicherungsverwahrung allerdings kaum behaupten können (vgl. zusammenfassend z. B. Kinzig, Die Sicherungsverwahrung auf dem Prüfstand, 1996). Einmal mehr wird deutlich, dass es trotz jahrzehntelanger Bemühungen einzelner Forscher und Forschergruppen über die Rechtswirklichkeit des Straf- und Maßregelvollzugs nach wie vor an grundlegenden Erkenntnissen fehlt (vgl. Dünkel, Empirische Forschung im Strafvollzug, 1996, S. 44 ff.). Wie wichtig eine kritische Begleitung des Strafvollzugs nicht zuletzt auch durch die Rechtsprechung ist, zei- 
gen die Hinweise auch in der vorliegenden Entscheidung, dass der Vollzug seine zunehmend restriktive Lockerungspraxis überdenken muss. In den erwähnten Entscheidungen von 1998 ging es ebenfalls um Gefangene mit sehr langen Inhaftierungszeiten (u. a. Lebenslängliche), bei denen mit zunehmender Vollzugsdauer angesichts der möglichen bedingten Entlassung dem Vollzug die Pflicht erwächst, die prognostischen Entscheidungsgrundlagen durch eine verantwortungsbewusste Lockerungspraxis zu verbessern (vgl. z. B. BVerfG ZfStrVo 1998, 180; NStZ 1998, 373; NStZ 1998, 430; NJW 1998, 1133; NStZRR 1998, 121; vgl. aber auch die Lockerungen im Einzelfall ausschließenden Entscheidungen in BVerfG NStZ 2002, 222 bzgl. eines zur Mitarbeit am Vollzugsziel nicht bereiten Lebenslänglichen, und zwa trotz 26-jähriger Verbüßungszeit, und BVerfG 2 BvR 116/02 vom 12.6.2002 bzgl. eines Sicherungsverwahrten auch nach mehr als 10jähriger Vollstreckung).

Im übrigen wird man mit dem kriminalpolitischen Ergebnis der Entscheidung insofern leben können, als das BVerfG klargestellt hat, dass es hinsichtlich der Fortdauer über die früher absolut geltende Zehnjahresfrist hinaus i. S. einer faktischen Beweislastumkehr des Nachweises der fortbestehenden Gefährlichkeit bedarf, der sich in der Praxis auf die wenigen (möglicherweise wirklich gefährlichen) Einzelfälle beschränken wird.

\section{Kompetenzfragen}

Die zweite $\mathrm{zu}$ besprechende Entscheidung betrifft die Frage der landesrechtlichen Gesetze bzgl. einer nachträglichen Sicherungsverwahrung. Das »Bayerische Gesetz zur Unterbringung von besonders rückfallgefährdeten hochgefährlichen Straftätern« vom 24.12.2001 ebenso wie das "Gesetz des Landes Sachsen-Anhalt über die Unterbringung besonders rückfallgefährdeter Personen zur Abwehr erheblicher Gefahren für die öffentliche Sicherheit und Ordnung « vom 6.3.2002 sehen die nachträgliche Anordnung der Sicherungsverwahrung in Fällen vor, bei denen die formellen Voraussetzungen der Sicherungsverwah- rung des $\S 66$ Abs. 1 Nr. 1 und 2, Abs. 2 bis 4 StGB zum Urteilszeitpunkt vorlagen und »aufgrund von Tatsachen, die nach der Verurteilung eingetreten sind, davon auszugehen ist, dass von dem Betroffenen eine gegenwärtige erhebliche Gefahr für das Leben, die körperliche Unversehrtheit, die Freiheit der Person oder die sexuelle Selbstbestimmung anderer ausgeht, insbesondere weil er im Vollzug der Freiheitsstrafe beharrlich die Mitwirkung an der Erreichung des Vollzugsziels (§ 2 des Strafvollzugsgesetzes) verweigert, namentlich eine rückfallvermeidende Psycho- oder Sozialtherapie ablehnt« (Art. 1 Abs. 1 BayStrUBG; nahezu wörtlich gleichlautend Art. 1 Abs. 1 UBG LSA). Während in Bayern die von der Strafvollstreckungskammer in der Besetzung mit drei Berufsrichtern ergehende Entscheidung grundsätzlich unbefristet ist, gilt die Entscheidung in Sachsen-Anhalt zunächst nur für 6 Monate, mit der Möglichkeit einer Verlängerung um weitere 12 Monate.

Das BVerfG stellt in seiner Entscheidung vom 5.2.2004 die Verfassungswidrigkeit der beiden Gesetze wegen Verstoßes gegen Art. 74 Abs. 1 in Verbindung mit Art. 70 Abs. 1 und Art. 72 Abs. 1 GG fest. Es betrachtet die landesrechtlichen Regelungen materiell als Strafrecht, weil eine »an Wortlaut, Gesetzesgeschichte, Systematik und Normzweck orientierte Auslegung ergibt, dass zum Strafrecht die Regelung aller, auch nachträglicher, repressiver oder präventiver staatlicher Reaktionen auf Straftaten gehört, die an die Straftat anknüpfen, ausschließlich für Straftäter gelten und ihre sachliche Rechtfertigung auch aus der Anlasstat beziehen. « Nach dieser weiten Auffassung von »Strafrecht « fallen darunter alle Strafen, Bußen oder präventive Reaktionen wie Maßregeln der Besserung und Sicherung, die als Reaktion für eine rechtswidrige Straftat vorgesehen sind. Der Sachzusammenhang zum Strafrecht bestehe auch noch, wenn - wie im Fall des § 66a StGB - erst nachträglich über die im Urteil vorbehaltene Sicherungsverwahrung entschieden werde. Dies mache $\S 66$ a Abs. 2 StGB deutlich, wo auf die Gesamtwürdigung des Verurteilten, seiner Taten und der Entwicklung im Vollzug abgestellt werde.
Bei den angegriffenen landesgesetzlichen Regelungen sei in vergleichbarer Weise dieser Sachzusammenhang zum Strafrecht hergestellt, denn die Unterbringung sei auf Straftäter begrenzt und knüpfe an bestimmte Anlasstaten an. Das BVerfG weist in diesem Zusammenhang ausdrücklich darauf hin, dass eine straftatenunabhängige Unterbringung (z. B. auch strafrechtlich nicht Verurteilter) jenseits der landesgesetzlich geregelten Unterbringung psychisch Kranker unter dem Gesichtspunkt der Verhältnismäßigkeit nicht vertretbar wäre. Die »längerfristige Verwahrung eines psychisch gesunden und strafrechtlich nicht oder nur unerheblich vorbelasteten Bürgers zum Zweck einer von ihm ausgehenden Gefahr der Begehung von Straftaten « wäre mit dem GG »nicht vereinbar.«

Ebenso wenig wäre eine von der Anlasstat losgelöste Prognosestellung bei der nachträglichen Anordnung einer Unterbringung verfassungsrechtlich nicht zulässig. Das BVerfG spricht insoweit von einem »misslungenen Kunstgriff « der Lan- lücke schließen wollten, so könne die behauptete Lücke kompetenzrechtlich nicht anders beurteilt werden als das lückenhafte Gesetz, d. h. als dem Strafrecht zuzuordnende Materie.

Die Verfassungswidrigkeit der beiden Landesgesetze folgt daraus, dass der Bundesgesetzgeber die Materie der Sicherungsverwahrung umfassend und damit abschließend im Sinne des Art. 72 Abs. 1 GG regeln wollte und auch geregelt hat, so dass den Ländern insoweit keine Gesetzgebungskompetenz verblieb.

Erstaunlicherweise führt die Verfassungswidrigkeit der beiden Gesetze allerdings nicht zu ihrer Nichtigkeit. Vielmehr hat das BVerfG ihre Weitergeltung bis zum 30.9.2004 angeordnet. »Dies komme dann in Betracht, wenn die sofortige Ungültigkeit ... dem Schutz überragender Güter des Gemeinwohls die Grundlage entziehen würde und eine Abwägung mit den betroffenen Grundrechten ergibt, dass der Eingriff für eine Übergangszeit

\section{"Die Sicherungsverwahrung dient im Gegensatz zur Strafe nicht dem Zweck, begangenes Unrecht zu sühnen, sondern dazu, die Allgemeinheit vor dem Täter zu schützen.«}

desgesetzgeber (mit dem die polizeirechtliche Natur der Materie belegt werden sollte). Vielmehr sei es angesichts der Schwere des Eingriffs verfassungsrechtlich geradezu geboten, bei der Prognose auf »sämtliche entscheidungserheblichen Daten aus Lebens- und Kriminalitätsgeschichte des Betroffenen, einschließlich der begangenen Straftaten, « zurückzugreifen.

Die Zuordnung der landesgesetzlich geregelten Materie ergibt sich nach Auffassung des BVerfG auch daraus, dass die Detailvorschriften zum Verfahren den für die Sicherungsverwahrung geltenden Regelungen entsprechen. Wenn die Landesgesetzgeber - wie aus der Begründung der Gesetze zu entnehmen sei - eine bestehende Gesetzes- hinzunehmen ist." Da nach den in den vorliegenden Fällen übereinstimmenden mehreren Gutachten von einer hochgradigen Gefährlichkeit der Untergebrachten ausgegangen werden müsse, habe die Abwägung zu einem Vorrang des Schutzes des Gemeinwohls geführt. In diesem Zusammenhang sieht das BVerfG das materielle Anliegen der beiden Gesetze als in extremen Einzelfällen legitimierbar an, äußert sich allerdings angesichts des gesetzgeberischen Gestaltungsspielraums nicht dazu, wie diese Regelungen verfassungsrechtlich einwandfrei zu gestalten wären. Ein vom Bundesgesetzgeber entwickeltes Konzept nachträglicher Anordnung einer präventiven Verwahrung noch inhaftierter Straftäter stehe »bei entsprechend enger Fassung nicht von 
vornherein unter dem Verdikt der Verfassungswidrigkeit."

Der Entscheidung ist hinsichtlich der kompetenzrechtlichen Frage uneingeschränkt zuzustimmen, bzgl. der Fortgeltung der verfassungswidrigen Gesetze dagegen nicht. Dass es sich bei den landesrechtlichen Regelungen um ein missglücktes kriminalpolitisches Manöver gehandelt hat, den zu Recht zögerlichen Bundesgesetzgeber zu umgehen, war im Schrifttum von verschiedener Seite bereits zuvor dargelegt worden (vgl. u. a. Dünkel/Kunkat, Neue Kriminalpolitik 2001, 16 f.; Kinzig NJW 2001, 1455 ff.; Ullenbruch NStZ 2001, 292 ff.). Insofern ist der oben erwähnten Begründung des BVerfG nichts hinzuzufügen.

Problematischer gestaltet sich dagegen der zweite Teil der Entscheidung, in dem das überwiegende Interesse des Gemeinwohls eine vorübergehende Fortgeltung der Gesetze festlegt und damit die weitere Inhaftierung der beiden Betroffenen in Bayern und Sachsen-Anhalt ermöglicht.

Die Entscheidung insoweit erging mit $5: 3$ Stimmen und es lohnt sich, einen Blick auf das Votum der drei abweichenden Richter Broß, Osterloh und Gerhardt zu werfen. Sie sehen ein verfassungsrechtliches $\mathrm{Ge}$ bot der Fortgeltung nur, wenn der Gesetzgeber es unter Verletzung des Untermaßverbots überhaupt versäumt habe, für einen ausreichenden Schutz von Leib und Leben, persönlicher Freiheit etc. zu sorgen, oder wenn er gänzlich ungeeignete Regelungen getroffen habe. Davon könne hier keine Rede sein, zumal mit der im Falle der Entlassung eintretenden Führungsaufsicht (vgl. §§ 67d Abs. 2, 3 und 5, 68f StGB) ein eingriffsintensives Instrument der Kontrolle und Hilfe existiere. Verstöße gegen Weisungen im Rahmen der Führungsaufsicht sind strafbewehrt (vgl. § 145a StGB), ferner können mit den Weisungen auch Maßnahmen der Gefahrenabwehr nach allgemeinem Polizeirecht verbunden werden (z. B. verdeckte und offene Observationen). Unter Umständen wird auch eine Unterbringung nach den nach wie vor uneingeschränkt geltenden Landesgesetzen bzgl. der Unterbringung psychisch Kranker $\mathrm{zu}$ bedenken sein.
Die verschiedenen Netzwerke sozialer Kontrolle verdeutlichen, dass im Falle der Entlassung der Betroffenen die Gesellschaft nicht schutzlos gestellt wäre. Zudem verweist das abweichende Votum darauf, dass mit der Nichtigkeit der Landesgesetze der Rechtszustand, der vor ihrem Erlass bestand, wiederhergestellt würde, der dem Willen des Bundesgesetzgebers entspricht, der 1998 und 2002 zwar eine Ausweitung der Sicherungsverwahrung beschlossen habe, sich jedoch gegen weitergehende Verschärfungen, wie sie die Landesgesetze durchsetzten wollten, ausgesprochen habe. Dies ergebe sich schon aus der auch von der Senatsmehrheit festgestellten Tatsache, dass der Bundesgesetzgeber die Materie umfassend und abschließend regeln wollte bzw. geregelt habe. »Hat der Bund sein Gestaltungsermessen bereits rabschließend ausgeübt, so ist es nicht Aufgabe des Bundesverfassungsgerichts, ihm ein solches unter Verletzung des Freiheitsgrundrechts der Beschwerdeführer offen zu halten. Dass der Gesetzgeber hier irrtümlich von der Zuständigkeit der Länder ausging, ändert nichts daran, denn der Bund hat sich bei den Novellierungen 1998 und 2002 zur Sicherungsverwahrung in der Tat auch inhaltlich mit weitergehenden Forderungen der Länder befasst, sie aber nicht für notwendig bzw. verfassungsrechtlich haltbar angesehen (s. u. V.). Wenn das BVerfG sich über diese Entscheidung hinwegsetze und eigene rechtspolitische Wertungen, die nur dem Gesetzgeber zustehen, vornehme, so verstoße es damit gegen das Prinzip der Gewaltenteilung. Folgerichtig gelangen die abweichenden Richter zum Schluss, dass es für die weitere Unterbringung der Beschwerdeführer an einer gesetzlichen Grundlage fehle. Ein weiteres Argument gegen die Weitergeltung der Landesgesetze wird aus dem Rückwirkungsverbot des Art. 2 Abs. 1 in Verbindung mit Art. 20 Abs. 3 GG entnommen. Hat der Betroffene seine Strafe verbüßt, so würde mit der Unterbringung aufgrund der Straftäterunterbringungsgesetze in Anbetracht der Anknüpfung an die frühere Anlasstat eine echte Rückwirkung bezogen auf bereits abgeschlossene Sachverhalte geschaffen. Diese Durchbrechung der Rechtskraft ist aber nur unter den engen Voraussetzungen des $§ 362$ StPO vorgesehen und verfassungsrechtlich möglich.

Das abweichende Votum überzeugt mehr als die Mehrheitsmeinung, weil es die vielfältigen Möglichkeiten der strafrechtlichen, polizeirechtlichen und allgemeinen Sozialkontrolle stärker berücksichtigt, ohne zu verkennen, das es sich im vorliegenden Fall möglicherweise um tatsächlich sehr gefährliche Personen handelt. Diese Personen gab es jedoch immer schon und es ist nicht ersichtlich, dass die Gesellschaft völlig hilflos gegenüber entsprechenden Tätern kapitulieren musste. Harsche Kritik hat eingestuften Gesetze bis zum 30.9.2004 wurde - wie erwähnt damit begründet, dass dem Bundesgesetzgeber die Möglichkeit gegeben werden solle, die als Landesgesetz unzulässigen Regelungen bundesgesetzlich $\mathrm{zu}$ novellieren. Dementsprechend hat das Bundesjustizministerium bereits am 9.3.2004 einen Referentenentwurf vorgelegt, der die nachträgliche Unterbringung in der Sicherungsverwahrung auch für Fälle vorsieht, in denen sie im Urteil nicht vorbehalten wurde.

Darin wird ein $\S 66 \mathrm{~b}$ StGB zur nachträglichen Unterbringung in

Die Verfassungswidrigkeit der beiden Landesgesetze folgt daraus, dass der Bundesgesetzgeber die Materie der Sicherungsverwahrung umfassend und damit abschließend im Rahmen des zweispurigen Sanktionensystems regeln wollte und auch geregelt hat, so dass den Ländern insoweit keine Gesetzgebungskompetenz verblieb.

dementsprechend auch der frühere BGH-Richter Foth geäußert, der die Fortgeltung der Landesgesetze trotz Verfassungswidrigkeit für »unfassbar, ja sogar für strafbar « hält (vgl. Frankfurter Rundschau vom 25.2.2004, 4). Das BVerfG hätte die sofortige Freilassung der Betroffenen anordnen müssen, ansonsten läge ein Fall von Freiheitsberaubung vor (dementsprechend müsse die Staatsanwaltschaft diesbezüglich ermitteln). Ein verfassungswidriges freiheitsentziehendes Gesetz könne (im Gegensatz etwa zu verfassungswidrigen Steuergesetzen o. ä.) »nicht einen Tag« weitergelten.

Diese drastische Wortwahl eines der ranghöchsten (ehemaligen) Richters mag überraschen, ist jedoch konsequent, wenn man der auch im Minderheitsvotum dargelegten Meinung folgt. Es wird interessant sein zu verfolgen, wie sich die vorliegenden Fälle weiter entwickeln.

V.

Die zu Recht kritisierbare (s. o.) Befristung der als verfassungswidrig der Sicherungsverwahrung vorgeschlagen. § 66b Abs. 1 soll wie folgt lauten: »Werden nach einer Verurteilung wegen einer der in $\S 66$ Abs. 3 Satz 1 genannten Straftaten « (d. h. im Falle von Sexualdelikten) "vor Ende des Vollzugs der Freiheitsstrafe Tatsachen erkennbar, die auf eine erhebliche Gefährlichkeit des Verurteilten für die Allgemeinheit hinweisen, so kann das Gericht die Unterbringung in der Sicherungsverwahrung nachträglich anordnen, wenn die Gesamtwürdigung des Verurteilten, seiner Taten, und seiner Entwicklung während des Strafvollzugs ergibt, dass er mit hoher Wahrscheinlichkeit erhebliche Straftaten begehen wird, durch welche die Opfer seelisch oder körperlich schwer geschädigt werden, und wenn die übrigen Voraussetzungen des $\S 66$ Abs. 3 erfüllt sind.«

$\S$ 66b Abs. 2 erweitert den Anwendungsbereich auf andere Gewalttaten als Anlasstaten, wenn eine Verurteilung $\mathrm{zu}$ mindestens vier Jahren Freiheitsstrafe vorliegt. Schließlich soll nach Abs. 3 die nachträgliche Sicherungsverwahrung auch bei aus der Maßregel der 
Unterbringung in einem psychiatrischen Krankenhaus gem. § 63 StGB zu Entlassenden möglich sein, wenn zwar die schuldausschließenden Gründe entfallen sind, jedoch in Verbindung mit erheblichen Vorstrafen (Vorverurteilung zu mindestens drei Jahren oder frühere Unterbringung) und Anlasstaten i. S. d. § 66 Abs. 3 eine entsprechende Gefährlichkeit besteht.

Nachdem durch das Gesetz zur Änderung der Vorschriften über die Straftaten gegen die sexuelle Selbstbestimmung vom 19.12.2003 (BGBl. I, 3007; vgl. hierzu Frommel, Neue Kriminalpolitik 2004, 6 ff.) bereits die vorbehaltene Sicherungsverwahrung des § 66a StGB auf nach Erwachsenenstrafrecht abgeurteilte Heranwachsende ausgedehnt wurde (vgl. § 106 Abs. 3 JGG), will der vorliegende Referentenentwurf auch hier weitere Verschärfungen anbringen und die »echte " nachträgliche Sicherungsverwahrung des $\S 66 \mathrm{~b}$ StGB-E bei Heranwachsenden bei einer Verurteilung bzgl. der Anlasstat von mindestens 5 Jahren Freiheitsstrafe vorsehen. Weiterhin soll die nachträgliche Sicherungsverwahrung bei aus dem psychiatrischen Maßregelvollzug Entlassenen unter den o. g. Umständen ermöglicht werden (vgl. § 106 Abs. 5 und 6 JGG-E).

Das Verfahren soll nach den Vorschriften der StPO in Form eines erneuten Hauptverfahrens stattfinden (vgl. § 275a StPO-Entwurf). Voraussetzung der Anordnung sollen zwei Gutachten von externen (nicht mit der Behandlung des Gefangenen oder Untergebrachten befassten) Sachverständigen sein.

Unter Gesichtspunkten der Rückwirkung von Bedeutung ist, dass nach Art. 1a EGStGB des Entwurfs das Gesetz auch für alle derzeit nach Landesrecht in Baden-Württemberg, Bayern, Niedersachsen, Sachsen-Anhalt und Thüringen Untergebrachten gelten soll.

Mit dem vorliegenden Entwurf will der Gesetzgeber jede noch denkbare "Sicherheitslücke« schließen. Letztlich handelt es sich um die vollständige Übernahme der aus Kompetenzgründen verfassungswidrigen Landesgesetze.
Der Entwurf ist in verschiedener Hinsicht zu kritisieren und letztlich mit den vom BVerfG angedeuteten verfassungsrechtlichen Anforderungen nicht vereinbar.

Das »Einknicken« des Bundesjustizministeriums vor der konservativen Bundesratsmehrheit ist symptomatisch für eine weitgehend fehlende eigene kriminalpolitische Konzeption der derzeitigen rot-grünen Bundesregierung (vgl. zur Kritik bereits Dünkel, Neue Kriminalpolitik 2003, 2 f.). Mindestens genauso gravierend erscheint jedoch, dass die Bundesregierung den ihr vom BVerfG eingeräumten Gestaltungsspielraum nicht genutzt hat. Anstatt die Ländergesetze in Bundesrecht zu transformieren, hätte man ja durchaus Alternativen bedenken können. Man hätte zum einen die vom BVerfG geforderte »enge Ausnahmeregelung « tatsächlich eng fassen können und nur bei bestimmten Anlasstaten, wie vorsätzlicher Tötung und Vergewaltigung/sexueller Nötigung, vorsehen können. Man hätte statt auf »Wegsperren « auf die im Minderheitsvotum hingewiesenen ambulanten Formen der Kontrolle setzen und die personelle und sachliche Ausgestaltung der Führungsaufsicht qualitativ absichern können.

Nicht zuletzt verbleiben angesichts der engen Maßstäbe, die das $B$ VerfG andeutet, erhebliche verfassungsrechtliche Bedenken. Die vorliegende Regelung verstößt im Gegensatz zur (ebenfalls zweifelhaften, aber immerhin insoweit noch vertretbaren) vorbehaltenen Sicherungsverwahrung des $\S 66$ a StGB eindeutig gegen das Rückwirkungsverbot. Eine derartige Durchbrechung der Rechtskraft wäre allenfalls beim Nachweis anders nicht abwendbarer konkreter hoher Gefahren für das Gemeinwohlinteresse denkbar. Dieses wird zwar behauptet, jedoch werden die Alternativen einer verbesserten Infrastruktur, Behandlung, Aufsicht und Kontrolle innerhalb und außerhalb des Strafund Maßregelvollzugs nicht erwogen. Dies erscheint um so gravierender, als die nachträgliche Sicherungsverwahrung infolge des Verweises auf $\S 66$ Abs. 3 auch für Ersttäter und gem. § 106 Abs. 5, 6 JGG-E auch für Heranwachsende in Frage kommt. Damit wird trotz gegenteiliger Beteuerung ein in seiner Weite unverhältnismäßiges Instrument geschaffen.

Auch wenn das BVerfG die Einwände aufgrund der Unwägbarkeiten der Prognosestellung nicht für durchgreifend hält, kommt diesem Sachverhalt hier dennoch eine auch verfassungsrechtlich bedeutsame Qualität zu: wie hoch muss die Treffsicherheit der Gefährlichkeitsprognose denn sein, um der »hohen Wahrscheinlichkeit « weiterer schwerster Straftaten zu genügen? 99\%, 95\%, 80\% oder gar weniger? Dabei sind zwei Dimensionen der Wahrscheinlichkeitsaussage zu unterscheiden: zum einen das "hohe Rückfallrisiko an sich, zum anderen bezogen gerade auf die besonders schweren Taten gegen das Leben, die körperliche Unversehrtheit etc. Letztere Frage dürfte schon deshalb außerordentlich schwierig zu beantworten sein, weil die generellen Basisraten der Rückfallwahrscheinlichkeit bei Gewalt- und Sexualtätern besonders niedrig sind (vgl. Nomos-Kommentar-Dünkel 2003, § 57 Rn. 110 ff., 123 ff.; Jehle/Heinz/ Sutterer, Legalbewährung nach strafrechtlichen Sanktionen, 2003, 69 ff.)

Diese prognostischen Fragen tangieren den Bestimmtheitsgrundsatz i. S. d. Art. 103 Abs. 2 GG. Denn es gälte nach der Neuregelung drei Prognosestellungen $\mathrm{zu}$ unterscheiden, von denen die beiden Negativprognosen nicht klar genug definiert sind: So wird im Falle einer positiven Prognose, wenn dies unter Berücksichtigung der Sicherheitsinteressen der Allgemeinheit verantwortet werden kann, die bedingte Entlassung nach der Hälfte oder zwei Dritteln der Strafe gem. § 57 StGB erfolgen. Im Falle einer Negativprognose erfolgt die volle Strafverbüßung, während im Falle einer (in welcher Form?, mit welchem Grad an Wahrscheinlichkeit?) besonders negativen Prognose die Anordnung der nachträglichen Sicherungsverwahrung möglich sein soll.

Ferner geht es um die Frage der Erforderlichkeit im Rahmen der allgemeinen Verhältnismäßigkeitsprüfung. Könnte man mit hoher Trefferquote die Wahrscheinlichkeit schwerster zukünftiger Straftaten vorhersagen, wäre die Erforderlichkeit eher zu bejahen (vgl.
Peglau ZRP 2000, 150). Dabei müsste jeweils auch festgestellt werden, dass das mildere Mittel der Führungsaufsicht in Verbindung mit Weisungen und polizeilichen Maßnahmen zur Gefahrenabwehr (z. B. Observation) mit hoher Wahrscheinlichkeit nicht einen gleichwertigen Opferschutz gewährleisten würde.

Hierbei spielt eine Rolle, dass die gesetzliche Regelung des Referentenentwurfs Fälle betrifft, bei denen die formellen Voraussetzungen der Sicherungsverwahrung i. S. d. § 66 Abs. 3 StGB gegeben waren, eine besondere Gefährlichkeit aber zu diesem Zeitpunkt nicht angenommen wurde und auch kein Vorbehalt im Sinne von $\S 66$ a StGB erfolgt ist. Worauf kann sich die Prognose nunmehr stützen, um eine Gefährlichkeit zu belegen? Doch nur allein auf das Vollzugsverhalten! Das BVerfG hat in seiner Entscheidung vom 5.2.2004 jedoch betont, dass es verfassungsrechtlich geradezu geboten sei, bei der Prognose auf »sämtliche entscheidungserheblichen Daten aus Lebens- und Kriminalitätsgeschichte des Betroffenen, einschließlich der begangenen Straftaten, « zurückzugreifen und dass allein aus dem Vollzugsverhalten eine entsprechende Gefährlichkeitsprognose nicht begründbar sei. Wenn nun aber die beim Urteil ja bekannten Lebensumstände und Straftaten nicht einmal einen Vorbehalt im Sinne von $\S 66$ a StGB begründen konnten, wie soll dann in wundersamer Wandlung eine Gefährlichkeitsprognose angesichts fehlender Mitarbeit am Resozialisierungsvollzug o. ä. (in Verbindung mit den offensichtlichen eine Negativprognose nicht begründenden Lebens- und Tatumständen) mit der geforderten hohen Sicherheit überhaupt möglich sein? Dies wäre Metaphysik und hat mit den Möglichkeiten einer seriösen Prognoseforschung nichts gemein. Eine verfassungsrechtlich haltbare Prognosestellung wäre nur unter dem Eingeständnis möglich, dass man sich bei der Urteilsfällung hinsichtlich zukünftiger Gefährlichkeit völlig getäuscht habe. Dieses Eingeständnis führt dann allerdings zu der Frage, mit welcher Sicherheit ein weiterer Irrtum denn jetzt ausgeschlossen werden kann. Es gleicht der Quadratur des Kreises, was das BVerfG im Hinblick auf die Progno- 
seanforderungen tatsächlich abverlangt. Gutachter können und dürfen sich auf ein solches Spiel nicht einlassen, weil sie sich mit der Behauptung, Prognosen mit hohen Trefferquoten auf der Basis neuer Erkenntnisse lediglich im Bereich des Vollzugsverhaltens, unglaubwürdig machen und gegen die Regeln der wissenschaftlich vertretbaren Prognoseforschung verstoßen (vgl. hierzu schon in anderem $\mathrm{Zu}$ sammenhang kritisch Schöch in Festschrift für Kaiser 1998; Nedopil, Forensische Psychiatrie, 2000, 248; Nomos-Kommentar-Dünkel 2003, $\S 57$ Rn. 118 ff.).

Dass aus dem Vollzugsverhalten allein keine zutreffenden Schlussfolgerungen gezogen werden können, wurde bereits an anderer Stelle ausgeführt (vgl. Dünkel/Kunkat Neue Kriminalpolitik 2001, S. 16 f.).

Der Fall, dass zum Urteilszeitpunkt eine erhebliche Gefährlichkeit nicht diagnostiziert werden kann, dann aber im Vollzugsverlauf wohl, ist - wie gezeigt - wissenschaftlich nicht begründbar. Kinzig (NJW 2001, S. 1457 f.) hat eindrucksvoll belegt, dass schon in der jetzigen Situation mehr als $60 \%$, möglicherweise mehr als 90\% der Sicherungsverwahrten vermutlich zu den sog. »falsch Positiven «, d. h. zu den unzutreffend als gefährlich diagnostizierten gehören. Diese Art von Prognosefehlern wird sich mit der Klientel des $\S 66 \mathrm{~b}$ StGB noch weiter erhöhen. Es gehört zum gesicherten Kenntnisstand der Strafvollzugsforschung, dass der Schluss von intramuralem Verhalten auf das Verhalten nach der Entlassung nur sehr bedingt möglich ist. Sog. renitentes Verhalten kann den Überlebenswillen des Gefangenen dokumentieren und prognostisch neutral oder positiv zu werten sein, unauffälliges Verhalten kann Ausdruck von Zweckanpassung ohne ernsthafte Änderungsbereitschaft sein, das eher eine negative Prognose rechtfertigen kann. Letztlich handelt es sich bei alledem um Folgen der Inhaftierung, also einen Bereich, für den der Strafvollzug selbst zumindest mitverantwortlich erscheint. Der Strafvollzug bescheinigt sich in diesen Fällen sein eigenes Versagen. Nicht der vermeintlich gefährliche Gefangene, sondern die Verantwortlichen des Strafvollzugs werden so zum Sicherheitsrisiko.

Die im Gesetzentwurf genannten Indizien, wie etwa Drohungen des zu Entlassenden oder intensive Kontakte zu einem gewaltbereiten Milieu (vgl. Begründung, S. 20), erscheinen in keiner Weise als empirisch abgesicherte Kriterien. Jeder Anstaltsleiter kennt vermutlich entsprechende Drohungen, die aus einer aktuellen Frustration der Haftsituation heraus entstehen und $\mathrm{zu}$ meist nicht (dauerhaft) ernsthaft gemeint sind. Das Indiz einer während der Haft begangenen weiteren Straftat erscheint insoweit gänzlich ungeeignet, als es entweder zu einer weiteren Verurteilung mit der dann ggf. möglichen originären Sicherungsverwahrung führt oder so wenig schwerwiegend erscheint, dass es auch die nachträgliche Sicherungsverwahrung nicht zulässig erscheinen lässt. Zu Recht verweist die Begründung darauf, dass die Ablehnung oder der Abbruch einer (Sozial-)Therapie allein keine ausreichende Grundlage ist und nur im Rahmen der geforderten »Gesamtwürdigung « eine Rolle spielen kann. In der Tat werden Sozialtherapieabbrecher häufiger rückfällig als Programmteilnehmer, die bis zum Ende durchgehalten haben, jedoch werden auch insoweit nur die wenigsten Entlassenen wegen schwerer Gewalttaten verurteilt (vgl. zusammenfassend Dünkel/ Drenkhahn in Bereswill/Greve, Hrsg., Forschungsthema Strafvollzug, 2001, S. 387 ff.). Das Argument wäre im übrigen so lange als zynisch anzusehen wie Wartezeiten (in Baden-Württemberg von bis zu einem Jahr) oder fehlende Behandlungskapazitäten den Zugang zur Sozialtherapie $z$. T. unmöglich machten. Therapieabbruch kann im übrigen im Einzelfall als durchaus positive Reaktion und Überlebensstrategie angesehen werden.

Die vermeintlich rechtsstaatliche Absicherung der Gefährlichkeitsprognose auf der Basis zweier Gutachter erweist sich ebenfalls als unzulänglich. Es ist kaum denkbar, dass die beiden geforderten externen Gutachter, die den Gefangenen, seine Entwicklung und die Vollzugsbedingungen nicht kennen und schlechter einschätzen können als etwa der Anstaltspsychologe/-psy- chiater, zu einer treffsicheren Gefährlichkeitsprognose gelangen können. Die richterliche Anordnungskompetenz kann ebenfalls leer laufen, denn welcher Richter wird gegen zwei Gutachter das Risiko auf sich nehmen, Vorwürfen ausgesetzt $\mathrm{zu}$ werden, erheblichen Straftaten Vorschub geleistet zu haben?

Die Unverhältnismäßigkeit des $\S 66$ b StGB-E ergibt sich noch aus einem anderen Gesichtspunkt: das geltende Recht geht davon aus, dass nach voller Strafverbüßung Entlassene unter Führungsaufsicht gestellt werden, um durch eine entspre- merkt: Diese von einzelnen Bundesländern wiederholt und auch bei den Beratungen zur Einführung der vorbehaltenen Sicherungsverwahrung i. S. des $\S 66 a$ StGB geforderte Variante (vgl. z. B. den Bundesratsentwurf BR-Drs. 507/02) war 2002 noch verworfen worden, weil eine Mehrheit des Bundestags die Grenze der verfassungsrechtlich zulässigen Erweiterung der Sicherungsverwahrung in dem bereits im Urteil festzustellenden Vorbehalt sah. Die darüber hinausgehende nachträgliche Sicherungsverwahrung ohne Vorbehalt wurde abgelehnt, weil sie

Die Entscheidung, das Landesrecht nicht sofort für nichtig zu erklären, erging mit 5 : 3 Stimmen. Die Minderheit verweist darauf, dass mit der im Falle der Entlassung eintretende Führungsaufsicht ein eingriffsintensives Instrument der Kontrolle und Hilfe existiere.

chende Hilfe und Kontrolle das Rückfallrisiko zu vermindern (vgl. $\S 68 \mathrm{f} \mathrm{StGB})$. Schon der Verstoß gegen Weisungen und Auflagen ist wie erwähnt - strafbewehrt (§ 145a StGB). Der Entwurf für die nachträgliche Sicherungsverwahrung hat die Unzulänglichkeit der Führungsaufsichtspraxis weder behauptet, geschweige denn belegt. Wenn das mildere Mittel aber nicht offensichtlich weniger effizient erscheint, fehlt es für den einschneidenderen Eingriff in die Freiheitsrechte des betroffenen potenziellen Straftäters an der Erforderlichkeit. Diese wird auch im Einzelfall kaum belegbar sein, solange keine experimentell kontrollierten Wirkungsstudien in diesem Bereich vorliegen.

\section{Ein Pyrrhusieg der Gegner der nachträglichen Sicherungsver- wahrung?}

Das Urteil des BVerfG vom 5.2.2004 könnte sich nach alledem als Pyrrhusieg der Gegner der nachträglichen Sicherungsverwahrung erweisen. Denn der Bundesgesetzgeber scheint nunmehr bereit, dem Druck der Länder auch in diesem Bereich nachzugeben. Wohlge- eine Durchbrechung der materiellen und formellen Rechtskraft des Urteils bedeute, die rechtsstaatlichen Garantien zur Wiederaufnahme des Verfahrens zuungunsten des Verurteilten unterlaufe und gegen das allgemeine Rückwirkungsverbot des Art. 103 Abs. 2 GG verstoße. An diesen Bedenken hat sich nichts geändert und leider hat es das BVerfG versäumt, die verfassungsrechtlichen Grenzen einer allgemeinen nachträglichen Sicherungsverwahrung genauer abzustecken. Möglicherweise ist sie nämlich verfassungsrechtlich überhaupt nicht zulässig, auch wenn das BVerfG dieses Verdikt "nicht von vornherein « aussprechen wollte. Es wird also mit Sicherheit zu weiteren Verfassungsklagen kommen, falls der Gesetzentwurf nicht doch noch scheitert. Dies erscheint aber eher unwahrscheinlich, nachdem in den Reihen der SPD angesichts der anstehenden Walkämpfe und der medial angeheizten Debatte um Sicherheitslücken und -risiken kaum Widerstand zu erwarten ist. Einzige Hoffnung bleibt insoweit die Partei BÜNDNIS 90/DIE GRÜNEN, bei denen es immerhin bzgl. der Einbeziehung von Ersttätern und Heranwachsenden Bedenken gibt. 